\title{
Lepton non-universality in $B$ decays and fermion mass structure
}

\section{B. Grinstein, ${ }^{a}$ S. Pokorski ${ }^{b}$ and G.G. Ross ${ }^{c}$}

\author{
${ }^{a}$ Department of Physics, University of California San Diego, \\ 9500 Gilman Dr, La Jolla CA, 92093, U.S.A. \\ ${ }^{b}$ Institute of Theoretical Physics, Faculty of Physics, University of Warsaw, \\ ul. Pasteura 5, PL-02-093 Warsaw, Poland \\ ${ }^{c}$ Rudolf Peierls Centre for Theoretical Physics, Clarendon Laboratory, University of Oxford, \\ Parks Road, Oxford OX1 3PU, U.K. \\ E-mail: bgrinstein@ucsd.edu, pokorski@fuw.edu.pl, \\ g.ross1@physics.ox.ac.uk
}

ABSTRACT: We consider the possibility that the neutral-current $B$ anomalies are due to radiative corrections generated by Yukawa interactions of quarks and leptons with new vector-like quark and lepton electroweak doublets and new Standard Model singlet scalars. We show that the restricted interactions needed can result from an underlying Abelian family symmetry and that the same symmetry can give rise to an acceptable pattern of quark and charged lepton masses and mixings, providing a bridge between the nonuniversality observed in the $B$-sector and that of the fermion mass matrices. We construct two simple models, one with a single singlet scalar in which the flavour changing comes from quark and lepton mixing and one with an additional scalar in which the flavour changing can come from both fermion and scalar mixing. We show that for the case the new quarks are much heavier than the new leptons and scalars the B anomalies can be due to box diagrams with couplings in the perturbative regime consistent with the bounds coming from $B_{s}-\bar{B}_{s}, K-\bar{K}$ and $D-\bar{D}$ mixing as well as other lepton family number violating processes. The new states can be dark matter candidates and, in the two scalar model with a light scalar of $O(60) \mathrm{GeV}$ and vector-like lepton of $O(100) \mathrm{GeV}$, there can be a simultaneous explanation of the $B$-anomalies, the muon anomalous magnetic moment and the dark matter abundance.

KeYwords: Beyond Standard Model, Heavy Quark Physics, Quark Masses and SM Parameters

ArXiv EPrint: 1809.01766 


\section{Contents}

1 Introduction 2

2 Box diagrams 3

3 Family symmetry and flavour changing in the fermion sector 5

3.1 Heavy vector-like quark and lepton couplings 5

3.2 Dark matter 6

3.3 Fermion mass and mixing structure 6

$\begin{array}{lll}4 & \text { Flavour changing in the scalar sector } & 7\end{array}$

4.1 Family symmetry properties of $\tilde{\Phi} \quad 7$

4.2 Scalar mixing 8

4.3 Scalar couplings in the mass eigenstate basis 8

$4.4 \quad b \rightarrow s \mu^{+} \mu^{-} \quad 9$

$\begin{array}{lll}\text { 4.4.1 } & B_{s}-\bar{B}_{s} \text { mixing } & 10\end{array}$

5 Phenomenological analysis $\quad 11$

$5.1 K_{L}-K_{S}$ mass difference and $D-\bar{D}$ mixing $\quad 11$

$\begin{array}{lll}5.1 .1 & \text { Single scalar model } & 11\end{array}$

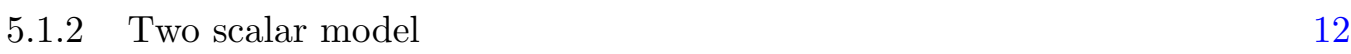

$5.2 B_{s}-\bar{B}_{s}$ mixing 12

$\begin{array}{lll}5.2 .1 & \text { Single scalar model } & 12\end{array}$

$\begin{array}{ll}5.2 .2 & \text { Two scalar model } \\ & 13\end{array}$

$5.3 \quad b \rightarrow s \mu \mu \quad 13$

$\begin{array}{lll}\text { 5.3.1 Single scalar model } & 14\end{array}$

$\begin{array}{ll}\text { 5.3.2 Two scalar model } & 14\end{array}$

$\begin{array}{ll}5.4 & \text { Anomalous magnetic moment of the muon } \\ \end{array}$

$\begin{array}{lll}5.4 .1 & \text { Single scalar model } & 15\end{array}$

$\begin{array}{ll}5.4 .2 & \text { Two scalar model } \\ \end{array}$

$\begin{array}{ll}5.5 \text { Comparison } & 16\end{array}$

$\begin{array}{ll}5.6 \mu \rightarrow e \gamma & 16\end{array}$

$\begin{array}{ll}5.7 Z \rightarrow \mu^{+} \mu^{-} & 17\end{array}$

6 Bounds on the new particle masses and their dark matter abundance 17

$\begin{array}{llr}7 & \text { Summary and conclusions } & 18\end{array}$

$\begin{array}{ll}\text { A Elimination of lepton flavour changing processes } & 19\end{array}$

$\begin{array}{ll}\text { B Simplified model without a dark matter candidate } & 20\end{array}$ 


\section{Introduction}

Recent measurements of $B$ decays [1-7] have indicated there may be departures from the Standard Model (SM) predictions for processes induced by the neutral current quark-level transition $b \rightarrow s \mu^{+} \mu^{-}$. Indeed an effective field theory analysis indicates that the inclusion of the operators ${ }^{1}$

$$
O_{9}=\frac{\alpha_{E M}}{4 \pi}\left[\bar{s} \gamma^{\nu} P_{L} b\right]\left[\bar{\mu} \gamma_{\nu} \mu\right], \quad O_{10}=\frac{\alpha_{E M}}{4 \pi}\left[\bar{s} \gamma^{\nu} P_{L} b\right]\left[\bar{\mu} \gamma_{\nu} \gamma_{5} \mu\right]
$$

that violate lepton universality can improve the global fit by $4-5 \sigma$ [8-13].

A departure from lepton universality is not really a surprise as non-universality in fermion masses and mixings is manifest. Given this it is relevant to ask whether the nonuniversal behaviour in B-decays may be related to the observed fermion mass structure. The most promising explanation for the origin of fermion masses and mixings is through a spontaneously broken family symmetry in which the order parameters for family symmetry breaking are the vacuum expectation values (vevs) of scalar (familon) fields, $\phi_{i}$, that transform non-trivially under the family symmetry. Then a particular entry in the fermion mass matrix is proportional to some power of the familon fields vevs, the underlying (higher dimensional) amplitude requiring the insertion of the familons to be family symmetry invariant. In the underlying theory the higher dimension amplitude is generated by the Yukawa couplings of SM fermions to the familons and new heavy vector-like fermions (the Froggatt-Nielsen mechanism [14]).

How could this be related to non-universality in B-decays? The neutral current process occurs first in the SM at one-loop order and is CKM suppressed. As a result the new physics responsible for non-universality must be small, consistent with a radiative correction involving new heavy states [15-19]. In particular it could be due to virtual processes involving heavy vectorlike fermions and familons associated with a family symmetry. Neutral current corrections involving loop effects of new scalars and fermions have already been explored in detail by Arnan, Hofer, Mescia and Crivellin [20] for a wide variety of SM representations for the scalars and quarks. ${ }^{2}$ Their overall conclusion is that to explain the $b \rightarrow s \mu^{+} \mu^{-}$anomaly the couplings required are forced to be uncomfortably large in comparison with the bounds coming from $B_{s}-\bar{B}_{s}$ mixing unless the scalars carry SM quantum numbers and the heavy fermions are Majorana fermions. This does not allow for a familon identification of the scalar in which case there is no obvious relation of the neutral current B-anomalies to the fermion mass structure.

In this paper we re-examine in detail the possibility that the anomalous $B$ neutral current decays can be due to new heavy vector-like quarks and leptons coupled to Standard Model states via new scalar fields. We show that, allowing for non-degenerate masses of these states, the constraints coming from $B_{s}-\bar{B}_{s}$ mixing are relaxed. We also show that the constraint can be further relaxed and indeed eliminated if the possibility of flavour

\footnotetext{
${ }^{1} P_{L, R}$ are the left- and right-handed projection operators.

${ }^{2}$ Neutral current corrections arising from loop effects in R-parity violating SUSY extensions of the SM have been investigated in ref. [21]. This approach does not lend itself to an interpretation as a theory of flavor.
} 
changing effects coming from the scalar sector is included. We investigate what type of family symmetry, capable of organising the quark and charged lepton masses and mixing, can generate the required structure without violating the many stringent constraints on flavour changing neutral currents. As we will see, this can be done but with a structure that is not normally considered when building fermion mass models.

Note that there is also an indication of a departure from SM predictions at the $3.9 \sigma$ level [42] in charged current processes induced by the quark-level transitions $b \rightarrow$ $c l \nu$ [22-31]. It is remarkable that it is possible to construct a simultaneous explanation of both charged and neutral current effects consistent with all present measurements, the most plausible being due to the exchange of new vector leptoquark states [32-41]. However, as the charged current processes arise at tree level, such non-universality cannot be explained by perturbative loop effects. Thus, in pursuing the familon explanation, we must assume that the signals for non-universality in charged current processes are spurious or that some other source of new physics is present.

In section 2 we briefly review the structure and calculation of the box diagrams, involving a heavy vector-like quark and lepton doublet together with a SM singlet scalar, give rise to $b \rightarrow s \mu^{+} \mu^{-}$and $B_{s}-\bar{B}_{s}$ mixing. Section 3 presents a simple family symmetry that generates the required coupling structure of the new states together with a realistic pattern of quark and charged lepton masses and mixing and the possibility that the new states are dark matter candidates. In section 4 we show how the addition of a second scalar field generates additional flavour changing effects coming from the scalar sector alone and calculate the associated box diagrams. In section 5 we present a phenomenological analysis of the resulting $B$ anomalies in both schemes concentrating on the case that the vector-like leptons and scalars are much lighter than the vector-like quark. We show that for couplings in the perturbative domain all neutral current anomalies can be accommodated consistent with the bounds on $B-\bar{B}, K-\bar{K}$ and $D-\bar{D}$ mixing. For the case that the vector-like lepton and the lightest scalar state are of $O(100) \mathrm{GeV}$, the SM prediction for the anomalous magnetic moment of the muon can also be brought into agreement with the experimental best fit value. We also discuss the constraints coming from other neutral flavour changing processes. In some of our flavour models there is a $Z_{2}$ symmetry under which SM fields are neutral; the lightest of the new particles that transforms non-trivially is therefore stable and a candidate for dark matter. In section 6 we discuss the experimental direct detection signals for the new particles, the present experimental limits and the dark matter abundance associated with the new states. Finally in section 7 we present our summary and conclusions. The appendices present two simple family symmetry variants capable of eliminating lepton flavour changing processes completely and allowing the lightest new state to decay so that it is no longer a dark matter candidate.

\section{Box diagrams}

Neutral current $B$ decays generated by scalars can proceed by the generic box diagram shown in figure 1(a). This involves two additional heavy vector-like quarks and leptons, $\Psi_{Q}$ and $\Psi_{\ell}$, and a scalar $\Phi$ with SM quantum numbers given in table 1 and interactions 


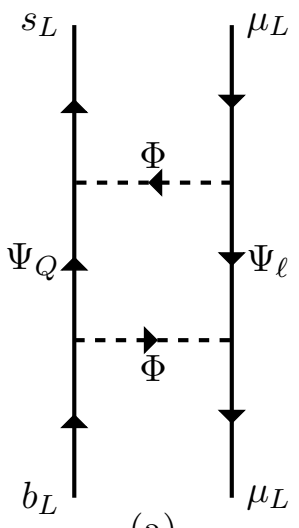

(a)

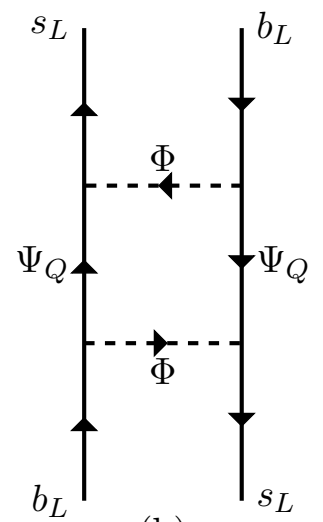

(b)

Figure 1. Box-diagrams contributing to (a) $b \rightarrow s \mu^{+} \mu^{-}$and (b) $B_{s} \rightarrow \bar{B}_{s}$, with couplings given in eq. (2.1).

\begin{tabular}{|c|ccc|}
\hline & $\mathrm{SU}(3)$ & $\mathrm{SU}(2)$ & $\mathrm{U}(1)$ \\
\hline$\Psi_{Q, L}$ & 3 & 2 & $1 / 6$ \\
$\Psi_{Q, R}$ & 3 & 2 & $1 / 6$ \\
$\Psi_{\ell, L}$ & 1 & 2 & $-1 / 2$ \\
$\Psi_{\ell, R}$ & 1 & 2 & $-1 / 2$ \\
$\Phi$ & 1 & 1 & 0 \\
\hline
\end{tabular}

Table 1. Multiplet structure of the additional states under $\mathrm{SU}(3) \times \mathrm{SU}(2) \times \mathrm{U}(1)$.

described by the Lagrangian

$$
\mathcal{L}_{\text {int }}=\sum_{i=d, s, b} \Gamma_{i}^{m} \bar{Q}_{i}^{m} P_{R} \Psi_{Q} \Phi+\sum_{i=e, \mu, \tau} \Gamma_{i}^{m, L} \bar{L}_{i}^{m} P_{R} \Psi_{\ell} \Phi+\text { h.c. }
$$

Here $Q_{i}^{m}$ and $L_{i}^{m}$ denote the SM left-handed quark and lepton doublet mass eigenstates with family index $i^{3}$ The effective Hamiltonian describing the $b \rightarrow s \mu^{+} \mu^{-}$transition has the form

$$
\mathcal{H}_{\text {eff }}^{\mu^{+} \mu^{-}}=C_{9} \mathcal{O}_{9}+C_{10} \mathcal{O}_{10}
$$

in terms of the operators given in eq. (1.1). The box diagram of figure 1(a) gives the Wilson coefficients

$$
C_{9}^{\text {box }}=-C_{10}^{\text {box }}=-\frac{\Gamma_{b \rightarrow s \mu^{+} \mu^{-}}}{32 \pi \alpha_{\mathrm{EM}} m_{\Phi}^{2}} F\left(x_{Q}, x_{\ell}\right)
$$

where $\Gamma_{b \rightarrow s \mu^{+} \mu^{-}}=\Gamma_{s}^{m} \Gamma_{b}^{m *}\left|\Gamma_{\mu}^{m}\right|^{2}$ and $x_{Q}=m_{\Psi_{Q}}^{2} / m_{\Phi}^{2}, x_{\ell}=m_{\Psi_{\ell}}^{2} / m_{\Phi}^{2}$, and the dimensionless loop function is given by

$$
F(x, y)=\frac{1}{(1-x)(1-y)}+\frac{x^{2} \log x}{(1-x)^{2}(x-y)}+\frac{y^{2} \log y}{(1-y)^{2}(y-x)}
$$

\footnotetext{
${ }^{3}$ We assume the upper components of the doublet $Q_{i}^{m}$ are linear combinations of the mass eigenstates, $V_{i j}^{\mathrm{CKM \dagger}} U_{j}$.
} 


\begin{tabular}{|c|ccccccccccccc|}
\hline & $Q_{2}$ & $q_{2}$ & $Q_{3}$ & $q_{3}$ & $L_{2}$ & $l_{2}$ & $L_{3}$ & $l_{3}$ & $\Psi_{Q,(L, R)}$ & $\Psi_{\ell,(L, R)}$ & $\Phi$ & $H$ & $\chi$ \\
\hline$Q_{F}$ & 2 & 0 & 0 & 0 & 2 & 1 & -2 & -2 & 2 & 4 & -2 & 0 & 1 \\
\hline
\end{tabular}

Table 2. $\mathrm{U}(1)_{F}$ family symmetry charges for the second and third families.

For the $B_{s}-\bar{B}_{s}$ mixing the Hamiltonian has the form

$$
\mathcal{H}_{\mathrm{eff}}^{B \bar{B}}=C_{B \bar{B}}\left(\bar{s} \gamma^{\mu} P_{L} b\right)\left(\bar{s} \gamma^{\mu} P_{L} b\right)
$$

where the box diagram, figure $1(\mathrm{~b})$, gives

$$
C_{B \bar{B}}=\frac{\Gamma_{B_{s}-\bar{B}_{s}}}{128 \pi^{2} m_{\Phi}^{2}} F\left(x_{Q}, x_{Q}\right)
$$

with $\Gamma_{B_{s}-\bar{B}_{s}}=\left(\Gamma_{s}^{m} \Gamma_{b}^{m *}\right)^{2}$.

\section{Family symmetry and flavour changing in the fermion sector}

In order to explain the anomaly it is necessary that the box diagram contributes principally to muon pair production, violating lepton universality. Given that the lepton masses also strongly violate lepton universality it is of interest to ask whether the two are related. Here we demonstrate that this can be done via a simple Abelian family symmetry, $\mathrm{U}(1)_{F}$, with the charge assignments given in table 2 . Here $Q_{i}$ and $L_{i}$ denote the left-handed (L) quark and lepton $\mathrm{SU}(2)$ doublets with family index $i, q_{i}$ and $l_{i}$ denote the right-handed (R) up and down quark and lepton $\mathrm{SU}(2)$ singlets, and $H$ is the Higgs doublet. We have also added a SM singlet scalar $\chi$ which acquires a vacuum expectation value (vev) and is responsible for generating the hierarchical structure of the quark and charged lepton masses and mixing.

\subsection{Heavy vector-like quark and lepton couplings}

With these charge assignments the only renormalisable couplings allowed involving the heavy vector-like states are given by

$$
\mathcal{L}_{\text {int }}=\Gamma_{b} \bar{Q}_{3} P_{R} \Psi_{Q} \Phi+\Gamma_{\mu} \bar{L}_{2} P_{R} \Psi_{\ell} \Phi+\text { h.c. }
$$

involving only the left-handed quark and lepton "current" eigenstates. The mass eigenstates, $Q_{i}^{m}, L_{i}^{m}$, in eq. (2.1) are superpositions of the current eigenstates, the mixing determined by mixing matrices $V^{Q}$ and $V^{L}$ for the up and down sectors:

$$
Q_{i}=\left(\begin{array}{c}
V_{i u}^{U} u+V_{i c}^{U} c+V_{i t}^{U} t \\
V_{i d}^{D} d+V_{i s}^{D} s+V_{i b}^{D} b
\end{array}\right), \quad L_{i}=\left(\begin{array}{c}
V_{i \nu_{1}}^{N} \nu_{1}+V_{i \nu_{2}}^{N} \nu_{2}+V_{i \nu_{3}}^{N} \nu_{3} \\
V_{i e}^{E} e+V_{i \mu}^{E} \mu+V_{i \tau}^{E} \tau
\end{array}\right), \quad i=2,3
$$

where the CKM and PMNS matrices are given by $V^{\mathrm{CKM}}=V^{U \dagger} V^{D}$ and $V_{P M N S}=V^{N \dagger} V^{E}$ respectively. Written in terms of the mass eigenstates, eq. (3.1) generates the Lagrangian in eq. (2.1). Note that the couplings $\Gamma_{b, \mu}$ to the current eigenstates can be of $O(1)$ while 
the coupling to mass eigenstates $\Gamma_{i}^{m}$ defined in the previous section include the mixing angles and can be small. In particular the relevant couplings in eq. (2.1) are given by ${ }^{4}$ $\Gamma_{s}^{m}=\Gamma_{b} V_{3 s}^{D} \approx \Gamma_{b} V_{c b}^{\mathrm{CKM}}$ and $\Gamma_{b}^{m}=\Gamma_{b} V_{3 b}^{D} \approx \Gamma_{b}$ and so the combination $\Gamma_{s}^{m *} \Gamma_{b}^{m} \propto V_{c b}^{\mathrm{CKM}}$ is small.

\subsection{Dark matter}

It is of interest to ask if the new states could be the source of dark matter. For this to be the case it is necessary for the lightest state to be stable and this can readily be arranged through an additional $Z_{2}$ symmetry under which only the fields $\Psi_{Q, \ell}, \Phi$ are odd. Then these states can only be produced in pairs and the lightest of these states will be stable and a candidate for dark matter. The case there is no $Z_{2}$ symmetry is briefly discussed in appendix B.

\subsection{Fermion mass and mixing structure}

The $\mathrm{U}(1)_{F}$ symmetry also controls the masses and mixing angles of the quarks and charged leptons when the symmetry is spontaneously broken. Quark and lepton masses are generated via the terms allowed by the $\mathrm{U}(1)_{F}$ symmetry in the effective Lagrangian:

$$
\begin{aligned}
\mathcal{L}_{\mathrm{eff}}^{m} \approx & \bar{Q}_{3, L} q_{3, R} H+\bar{Q}_{3, L} q_{2, R} H+\bar{Q}_{2, L} q_{2, R} H\left(\frac{\chi}{M_{q}}\right)^{2}+\bar{Q}_{2, L} q_{3, R} H\left(\frac{\chi}{M_{q}}\right)^{2} \\
& +\bar{L}_{3, L} l_{3, R} H+\bar{L}_{2, L} l_{2, R} H \frac{\chi}{M_{q}}+\bar{L}_{2, L} l_{3, R} H\left(\frac{\chi}{M_{q}}\right)^{4}+\bar{L}_{3, L} l_{2, R} H\left(\frac{\chi^{\dagger}}{M_{q}}\right)^{3}+\text { h.c. }
\end{aligned}
$$

where we have suppressed the $O(1)$ coupling constants. Terms with $\Psi_{Q, L}$ replaced for $Q_{2}$ are forbidden by a $Z_{2}$ symmetry under which only the fields $\Psi_{Q, \ell}, \Phi$ are odd; see section 3.2. $M_{q}$ are mediator masses ${ }^{5}$ associated with additional vector-like states associated with the Froggatt Nielsen mechanism. ${ }^{6}$ The second generation quark masses are suppressed relative to the third generation masses by the factor $\epsilon^{2}=\left(\frac{\langle\chi\rangle}{M_{q}}\right)^{2}$ and to explain why this ratio is smaller in the up quark sector than in the down quark sector we need $M_{u}>M_{d}$. The same factor determines the mixing of the left-handed states giving $V_{2 b}^{D} \sim \frac{m_{s}}{m_{b}}, V_{2 t}^{U} \sim \frac{m_{c}}{m_{t}}$. Thus the dominant contribution to the $(2,3)$ element of the CKM matrix comes from the down quark sector giving $V_{c b}^{\mathrm{CKM}} \sim \epsilon^{2} \sim V_{2 b}^{D}=O\left(\epsilon^{2}\right) \sim \frac{m_{s}}{m_{b}} \sim 0.04$.

The ratio of charged lepton masses is given by $\frac{m_{\mu}}{m_{\tau}}=O(\epsilon)$. The charged lepton mixing matrices are strongly constrained by the bounds on lepton flavour violation. For example, the branching ratio for $\tau \rightarrow \mu+\gamma$ is less than $10^{-8}$ and an even stronger bound holds for $\mu \rightarrow e+\gamma$. As discussed in section 5.6, to avoid violating these bounds the charged lepton mixing angles must be very small. ${ }^{7}$ Here the choice of the $\mathrm{U}(1)_{F}$ lepton charges ensures the

\footnotetext{
${ }^{4}$ Here we assume the mixing is dominated by the down quark sector as is often the case in family symmetry models which relate the quark mass hierarchy to the quark mixing angles.

${ }^{5}$ For simplicity we take the down quark and lepton mediators to have equal masses as might be justified by an underlying stage of Grand Unification.

${ }^{6}$ We assume these are much heavier than the vector-like states, $\Psi_{Q, L}$ introduced in eq. (2.1) so they do not contribute significantly B decays and mixing.

${ }^{7}$ This means that the large mixing angles observed in neutrino oscillation must come from the neutrino sector.
} 


\begin{tabular}{|c|cccc|}
\hline & $Q_{1}$ & $q_{1}$ & $L_{1}$ & $l_{1}$ \\
\hline$Q_{F}$ & 3 & -1 & -4 & 1 \\
\hline
\end{tabular}

Table 3. $\mathrm{U}(1)_{F}$ family symmetry charges for the first family.

mixing between the tau and muon is strongly suppressed and is of $O\left(\epsilon^{4}\right)$. An additional $Z_{3}$ symmetry can eliminate charged lepton mixing completely as discussed in appendix A.

What about the mass structure for the light generation? A viable structure for the quark masses and mixing together with a strong suppression of the mixing between the lepton doublets can readily be obtained via the same $\mathrm{U}(1)_{F}$ family symmetry through the choice of charges given in table 3 , with the interactions

$$
\begin{aligned}
\mathcal{L}_{\text {eff }}= & \bar{Q}_{1} P_{R} \Psi_{Q} \Phi\left(\frac{\chi}{M_{q}}\right)^{3}+\bar{Q}_{1, L} q_{1, R} H\left(\frac{\chi}{M_{q}}\right)^{4}+\bar{Q}_{1, L} q_{2, R} H\left(\frac{\chi}{M_{q}}\right)^{3}+\bar{Q}_{2, L} q_{1, R} H\left(\frac{\chi}{M_{q}}\right)^{3} \\
& +\bar{L}_{1, L} \Psi_{\ell, R} \Phi\left(\frac{\chi \dagger}{M_{q}}\right)^{6}+\bar{L}_{1, L} l_{1, R} H\left(\frac{\chi \dagger}{M_{q}}\right)^{5}+\bar{L}_{1, L} l_{2, R} H\left(\frac{\chi \dagger}{M_{q}}\right)^{5}+\bar{L}_{2, L} l_{1, R} H\left(\frac{\chi}{M_{q}}\right)+\text { h.c. }
\end{aligned}
$$

In the quark sector the down quark mass is suppressed relative to the strange quark masse by the factor $\epsilon^{2}$ and the Cabibbo angle is of order $\epsilon \approx \sqrt{\left(\frac{m_{d}}{m_{s}}\right)}$ giving a realistic mass and mixing angle structure. The additional coupling of $Q_{1}$ to the vector-like quark will induce further lepton non-universal decays at $O\left(\epsilon^{3}\right)$, the leading one being $b \rightarrow d \mu \mu$. As is shown in appendix B these can be eliminated by an extension of the family symmetry.

In the charged lepton sector the electron mass is suppressed relative to the muon mass by the factor $\epsilon^{4}$ while the mixing between the muon and electron doublets is highly suppressed being also of $O\left(\epsilon^{4}\right)$.

\section{Flavour changing in the scalar sector}

The model introduced in eq. (3.1) generates the flavour change in the quark sector via the quark mixing of eq. (3.2). However it is also possible that the flavour change occurs in the scalar sector and to illustrate this we generalise the model slightly to include another Standard Model singlet scalar $\tilde{\Phi}$ with the interaction Lagrangian now given by

$$
\mathcal{L}_{\text {int }}=\Gamma_{b} \bar{Q}_{3} P_{R} \Psi_{Q} \Phi+\widetilde{\Gamma}_{s} \bar{Q}_{2} P_{R} \Psi_{Q} \tilde{\Phi}+\Gamma_{\mu} \bar{L}_{2} P_{R} \Psi_{\ell} \Phi+\text { h.c. }
$$

The inclusion of the scalar $\tilde{\Phi}$ has no implications for the fermion mass and mixing model introduced in section 3 , except for presence of the additional coupling $\bar{Q}_{1} \Psi_{Q}\left(\tilde{\Phi} \frac{\chi}{M_{q}}\right)$ in eq. (3.4).

\subsection{Family symmetry properties of $\tilde{\Phi}$}

The new coupling involving $\tilde{\Phi}$ is allowed by the $\mathrm{U}(1)_{F}$ and $Z_{2}$ family symmetries provided it is uncharged under $\mathrm{U}(1)_{F}$ and odd under $Z_{2}$. Indeed, it can be a real field or, if complex, ${ }^{8}$

\footnotetext{
${ }^{8}$ In what follows we assume that $\tilde{\Phi}$ is complex.
} 
there is an additional coupling allowed in eq. (4.1), namely $\widetilde{\Gamma}_{s}^{\prime} \bar{Q}_{2} P_{R} \Psi_{Q} \tilde{\Phi}^{*}$, corresponding to the second term with $\tilde{\Phi}$ replaced by $\tilde{\Phi}^{*}$. One can avoid this by extending the family symmetry to give $\tilde{\Phi}$ a family charge. If this is not the case box diagrams involving an incoming and outgoing $\tilde{\Phi}$ coupled to the same fermion line will have additional crossed graphs with the $\tilde{\Phi}$ lines exchanged. For the case $\tilde{\Phi}$ is real the two graphs cancel exactly. For the case $\tilde{\Phi}$ is complex the combined contribution is proportional to $\widetilde{\Gamma}_{s}-\widetilde{\Gamma}_{s}^{\prime} \cdot{ }^{9}$ The cancellation or partial cancellation of these graphs does not affect the order of magnitude estimate for the $b \rightarrow s \mu^{+} \mu^{-}$and $B_{s}-\bar{B}_{s}$ mixing amplitudes presented in section 4.3 but does significantly change the expectation, discussed below, for $K-\bar{K}$ and $D-\bar{D}$ mixing coming from the box diagram involving only $Q_{2}$ current quarks.

\subsection{Scalar mixing}

Since $\Phi \tilde{\Phi} \chi^{2}$ is an allowed scalar coupling we expect $\Phi$ and $\tilde{\Phi}$ to mix so that the light and heavy $\Phi_{L}$ and $\Phi_{H}$ scalar mass eigenstates are mixtures of the "current" scalar states $\Phi$ and $\tilde{\Phi}$

$$
\begin{aligned}
\Phi_{L} & =\cos (\theta) \Phi+\sin (\theta) \tilde{\Phi} \\
\Phi_{H} & =-\sin (\theta) \Phi+\cos (\theta) \tilde{\Phi}
\end{aligned}
$$

In this case the leading contribution to $b \rightarrow s \mu^{+} \mu^{-}$is given by figure 2. Because $\Phi$ and $\tilde{\Phi}$ couple to different quark current eigenstates there will be a contribution to $B-\bar{B}$ mixing and $b \rightarrow s \mu \mu$ even if the quark mixing angles are zero provided the scalar mass eigenstates are mixture of the current eigenstates. In the limit that $\Phi$ and $\tilde{\Phi}$ do not mix, the Lagrangian (4.1) exhibits two separate U(1) symmetries $\Phi \rightarrow e^{i \alpha} \Phi, Q_{3} \rightarrow e^{i \alpha} Q_{3}$, $L_{2} \rightarrow e^{i \alpha} L_{2}, \tilde{\Phi} \rightarrow e^{i \beta} \tilde{\Phi}$, and $Q_{2} \rightarrow e^{i \beta} Q_{2}$, and the flavor mixing is all due to the matrices $V^{D, U, N, E}$ in eq. (3.2). If these states are close in mass there will be a significant cancellation of the light and heavy contributions.

\subsection{Scalar couplings in the mass eigenstate basis}

To simplify the presentation, here we concentrate on the case of maximal mixing between $\Phi$ and $\tilde{\Phi},{ }^{10} \theta=\pi / 4$, where now $\Phi_{L, H}=(\Phi \pm \tilde{\Phi}) / \sqrt{2}$. In table 4 we give an example how the phenomenology changes when the states depart from maximal mixing.

For the case that $\Phi$ and $\tilde{\Phi}$ are complex fields with only the couplings of eq. (4.1), the coupling of the maximally mixed fields to mass eigenstates is given by

$$
\begin{aligned}
\mathcal{L}= & \frac{1}{\sqrt{2}}\left[\left(\Gamma_{b} V_{3 b}^{*}+\widetilde{\Gamma}_{s} V_{2 b}^{*}\right) \Phi_{L}+\left(\Gamma_{b} V_{3 b}^{*}-\widetilde{\Gamma}_{s} V_{2 b}^{*}\right) \Phi_{H}\right] \bar{b}_{L} \Psi_{Q} \\
& +\left[\left(\Gamma_{b} V_{3 s}^{*}+\widetilde{\Gamma}_{s} V_{2 s}^{*}\right) \Phi_{L}+\left(\Gamma_{b} V_{3 s}^{*}-\widetilde{\Gamma}_{s} V_{2 s}^{*}\right) \Phi_{H}\right] \bar{s}_{L} \Psi_{Q}+\text { h.c. }
\end{aligned}
$$

Here and below $V_{i j} \equiv V_{i j}^{D}$. With these couplings it is straightforward to determine the $B_{s}-\bar{B}_{s}$ mixing and $b \rightarrow s \mu \mu$ amplitudes for the maximally mixed two scalar model.

\footnotetext{
${ }^{9}$ We assume that, as in the models presented in section 3 and in the appendix, $\Phi$ carries a family charge and so does not lead to further crossed graphs involving the $\Phi$ vertex.

${ }^{10}$ Such maximal mixing is natural if the coefficient of the quadratic term mixing $\Phi$ and $\tilde{\Phi}$ is much greater than the difference of the coefficients of the quadratic terms in $\Phi$ and $\tilde{\Phi}$ separately.
} 


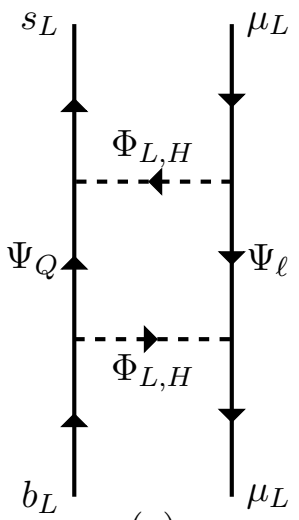

(a)

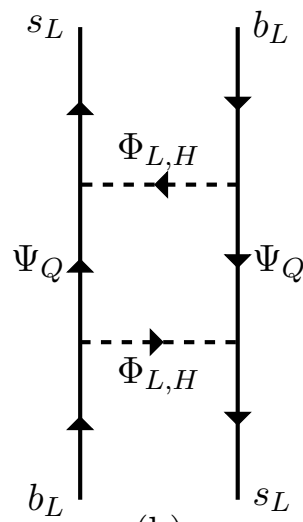

(b)

Figure 2. Box-diagrams contributing to (a) $b \rightarrow s \mu^{+} \mu^{-}$and (b) $B_{s} \rightarrow \bar{B}_{s}$, with couplings given in eq. (4.1).

\section{$4.4 \quad b \rightarrow s \mu^{+} \mu^{-}$}

The graph of figure 2(a) gives the Wilson coefficients

$$
\begin{aligned}
C_{9}= & -C_{10} \\
= & -\frac{1}{128 \pi \alpha_{E M} m_{\Psi_{Q}}^{2}}\left|\Gamma_{\mu} V_{2 \mu}^{E}\right|^{2}\left[\left(\Gamma_{b}^{*} V_{3 b}+\widetilde{\Gamma}_{s}^{*} V_{2 b}\right)\left(\Gamma_{b} V_{3 s}^{*}+\widetilde{\Gamma}_{s} V_{2 s}^{*}\right) G\left(y_{\ell}, y_{\phi_{L}}, y_{\phi_{L}}\right)\right. \\
& +2\left(\left|\Gamma_{b}\right|^{2} V_{3 b} V_{3 s}^{*}-\left|\widetilde{\Gamma}_{s}\right|^{2} V_{2 b} V_{2 s}^{*}\right) G\left(y_{\ell}, y_{\phi_{L}}, y_{\phi_{H}}\right) \\
& \left.+\left(\Gamma_{b}^{*} V_{3 b}-\widetilde{\Gamma}_{s}^{*} V_{2 b}\right)\left(\Gamma_{b} V_{3 s}^{*}-\widetilde{\Gamma}_{s} V_{2 s}^{*}\right) G\left(y_{\ell}, y_{\phi_{H}}, y_{\phi_{H}}\right)\right] \\
\approx & -\frac{1}{128 \pi \alpha_{E M} m_{\Psi_{Q}}^{2}}\left|\Gamma_{\mu} V_{2 \mu}^{E}\right|^{2}\left[\Gamma_{b}^{*} \widetilde{\Gamma}_{s}\left(G\left(y_{\ell}, y_{\phi_{L}}, y_{\phi_{L}}\right)-G\left(y_{\ell}, y_{\phi_{H}}, y_{\phi_{H}}\right)\right)\right. \\
& -V_{2 b}\left(\left|\Gamma_{b}\right|^{2}-\left|\widetilde{\Gamma}_{s}\right|^{2}\right)\left(G\left(y_{\ell}, y_{\phi_{L}}, y_{\phi_{L}}\right)+G\left(y_{\ell}, y_{\phi_{H}}, y_{\phi_{H}}\right)\right) \\
& \left.-2 V_{2 b}\left(\left|\Gamma_{b}\right|^{2}+\left|\widetilde{\Gamma}_{s}\right|^{2}\right) G\left(y_{\ell}, y_{\phi_{L}}, y_{\phi_{H}}\right)\right]
\end{aligned}
$$

In the last step we have used $\sum_{i} V_{i b} V_{i s}^{*}=0$ and $V_{i j} \approx V_{i j}^{\mathrm{CKM}}$ so that $V_{2 s} \approx V_{3 b} \approx 1$ and $V_{3 s} \approx-V_{2 b}^{*}$, with $\left|V_{2 b}\right| \sim 10^{-2}$.

The dimensionless function, $G$, is a function of three mass ratios $G \equiv G\left(y_{\ell}, y_{\Phi_{a}}, y_{\Phi_{b}}\right)$ with $y_{\ell}=m_{\Psi_{\ell}}^{2} / m_{\Psi_{Q}}^{2}, y_{\Phi_{a}}=m_{\Phi_{a}}^{2} / m_{\Psi_{Q}}^{2}, y_{\Phi_{b}}=m_{\Phi_{b}}^{2} / m_{\Psi_{Q}}^{2}$ given by ${ }^{11}$

$$
\begin{aligned}
G\left(y_{\ell}, y_{\Phi_{a}}, y_{\Phi_{b}}\right)= & \frac{y_{\ell}^{2} \ln \left(y_{\ell}\right)}{\left(y_{\ell}-1\right)\left(y_{\ell}-y_{\Phi_{a}}\right)\left(y_{\ell}-y_{\Phi_{b}}\right)}+\frac{y_{\Phi_{a}}^{2} \ln \left(y_{\Phi_{a}}\right)}{\left(y_{\Phi_{a}}-1\right)\left(y_{\Phi_{a}}-y_{\ell}\right)\left(y_{\Phi_{a}}-y_{\Phi_{b}}\right)} \\
& +\frac{y_{\Phi_{b}}^{2} \ln \left(y_{\Phi_{b}}\right)}{\left(y_{\Phi_{b}}-1\right)\left(y_{\Phi_{b}}-y_{\ell}\right)\left(y_{\Phi_{b}}-y_{\Phi_{a}}\right)}
\end{aligned}
$$

We see that there are two distinct contributions to $b \rightarrow s \mu^{+} \mu^{-}$. The term third line from the bottom of eq. (4.4) comes from the scalar mixing while the remaining terms,

\footnotetext{
${ }^{11}$ Note that $F(x, x) \equiv x^{-1} G\left(1, x^{-1}, x^{-1}\right)$.
} 
proportional to the small mixing angle $V_{3 b}$, come from quark mixing. To avoid the mixing angle suppression the contribution from scalar mixing should be large which in turn requires that the mass of $\Psi_{\ell}$ should not be significantly greater than mass difference, $\delta m$, between the masses of $\Phi_{H}$ and $\Phi_{L}$.

\subsection{1 $B_{s}-\bar{B}_{s}$ mixing}

The graph of figure $2(\mathrm{~b})$ gives

$$
\begin{aligned}
C_{B \bar{B}}= & \frac{1}{512 \pi^{2} m_{\Psi_{Q}}^{2}}\left[\left(\Gamma_{b}^{*} V_{3 b}+\widetilde{\Gamma}_{s}^{*} V_{2 b}\right)^{2}\left(\Gamma_{b} V_{3 s}^{*}+\widetilde{\Gamma}_{s} V_{2 s}^{*}\right)^{2} G\left(1, y_{\phi_{L}}, y_{\phi_{L}}\right)\right. \\
& +2\left(\left(\Gamma_{b}^{*} V_{3 b}\right)^{2}-\left(\widetilde{\Gamma}_{s}^{*} V_{2 b}\right)^{2}\right)\left(\left(\Gamma_{b} V_{3 s}^{*}\right)^{2}-\left(\widetilde{\Gamma}_{s} V_{2 s}^{*}\right)^{2}\right) G\left(1, y_{\phi_{L}}, y_{\phi_{H}}\right) \\
& \left.+\left(\Gamma_{b}^{*} V_{3 b}-\widetilde{\Gamma}_{s}^{*} V_{2 b}\right)^{2}\left(\Gamma_{b} V_{3 s}^{*}-\widetilde{\Gamma}_{s} V_{2 s}^{*}\right)^{2} G\left(1, y_{\phi_{H}}, y_{\phi_{H}}\right)\right]
\end{aligned}
$$

In the same limit discussed above this is approximately given by

$$
\begin{aligned}
C_{B \bar{B}} \approx & \frac{1}{512 \pi^{2} m_{\Psi_{Q}}^{2}}\left[\left(\Gamma_{b}^{*} \widetilde{\Gamma}_{s}\right)^{2}\left(G\left(1, y_{\phi_{L}}, y_{\phi_{L}}\right)+G\left(1, y_{\phi_{H}}, y_{\phi_{H}}\right)-2 G\left(1, y_{\phi_{L}}, y_{\phi_{H}}\right)\right)\right. \\
& -2 V_{2 b} \Gamma_{b}^{*} \widetilde{\Gamma}_{s}\left(\left|\Gamma_{b}\right|^{2}-\left|\widetilde{\Gamma}_{s}\right|^{2}\right)\left(G\left(1, y_{\phi_{L}}, y_{\phi_{L}}\right)-G\left(1, y_{\phi_{H}}, y_{\phi_{H}}\right)\right) \\
& +\left(V_{2 b}\right)^{2}\left[2\left(\left|\Gamma_{b}\right|^{2}-\left|\widetilde{\Gamma}_{s}\right|^{2}\right)^{2}\left(G\left(1, y_{\phi_{L}}, y_{\phi_{L}}\right)+G\left(1, y_{\phi_{H}}, y_{\phi_{H}}\right)\right)\right. \\
& \left.\left.-\left(\left|\Gamma_{b}\right|^{4}+\left|\widetilde{\Gamma}_{s}\right|^{4}\right)\left(G\left(1, y_{\phi_{L}}, y_{\phi_{L}}\right)+G\left(1, y_{\phi_{H}}, y_{\phi_{H}}\right)-2 G\left(1, y_{\phi_{L}}, y_{\phi_{H}}\right)\right)\right]+\cdots\right],
\end{aligned}
$$

where the ellipsis stand for terms of order $\left(V_{2 b}\right)^{3}$.

For the case $\delta m=m_{\Phi_{H}}-m_{\Phi_{L}} \ll m_{\Psi_{Q}}$ the first term, unsuppressed by powers of $V_{2 b}$, is suppressed by $\delta m^{2} / m_{\Psi_{Q}}^{2}$. The second term, suppressed by a single power of $V_{2 b}$, is also suppressed by $\delta m^{2} / m_{\Psi_{Q}}^{2}$ and by $\delta \Gamma / \Gamma=\left(\left|\Gamma_{b}\right|-\left|\Gamma_{s}\right|\right) /\left(\left|\Gamma_{b}\right|+\left|\Gamma_{s}\right|\right)$. Finally the terms on the third and fourth line of eq. (4.7), both suppressed by $V_{2 b}^{2}$, are additionally suppressed by $(\delta \Gamma / \Gamma)^{2}$ and $\delta m^{2} / m_{\Psi_{Q}}^{2}$, respectively. This demonstrates how in this limit the bound on $\Gamma_{B_{s}-\bar{B}_{s}}$ coming from $B_{s}-\bar{B}_{s}$ mixing does not significantly constrain $\Gamma_{b \rightarrow s \mu^{+} \mu^{-}}$; for example, in the combined limit $\delta m \rightarrow 0$ and $\delta \Gamma \rightarrow 0, C_{B \bar{B}}$ vanishes while $C_{9,10}^{\text {box }}$ remain of order $V_{2 b}$.

To summarise, for the case $m_{\Psi_{Q}} \gg m_{L} \sim m_{\Phi_{L}} \sim m_{\Phi_{H}}$, there are two main differences between the contributions coming from quark mixing and coming from scalar mixing. In the former the $b \rightarrow s \mu^{+} \mu^{-}$amplitude is suppressed by a factor of $V_{2 b}$ while in the latter there is no such overall suppression. Secondly, the relative suppression of the $B_{s}-\bar{B}_{s}$ mixing amplitude to the $b \rightarrow s \mu^{+} \mu^{-}$amplitude is $V_{2 b}$ in the former and $\delta m / m_{\Psi_{Q}}$ in the latter. Indeed combining the two contributions it is possible to eliminate the $B_{s}-\bar{B}_{s}$ mixing bound entirely. 


\section{Phenomenological analysis}

In this section we perform a numerical evaluation of the decay rates for the relevant processes and compare to the present measurements. For the case the box diagrams involve only a single scalar field, $\Phi$, all the flavour changing comes from quark mixing and we assume that this is dominated by the down quark sector. We will refer to this as the "Single scalar model".

For the case that the box diagrams involve both the $\Phi$ and $\tilde{\Phi}$ fields - the "Two scalar model" - the flavour changing effects come from both the down quark mixing and from the scalar mixing. To illustrate the expectation in this model we will usually present results for a benchmark point with the light scalar, $\Phi_{L}$, and the vector-like lepton having mass of $100 \mathrm{GeV}$ and the heavy scalar, $\Phi_{H}$, with mass of $400 \mathrm{GeV}$ assuming down quark mixing is dominant. To emphasize that the scalar mixing can be significant we also present results assuming that the down quark mixing is negligible together with the result assuming that the CKM mixing comes entirely from the down quark sector. Finally, to facilitate comparison between models, we quote the result for the benchmark choice, $\Gamma_{b}=1.5$.

For most observables the results are quite insensitive to the precise value of the light scalar mass. However, when discussing the dark matter and muon anomalous magnetic moment bounds, we will choose $m_{L}=105 \mathrm{GeV}$ and $m_{\Phi_{L}}=62 \mathrm{GeV}$ to compare to the detailed analysis of refs. [51, 52].

\section{1 $\quad K_{L}-K_{S}$ mass difference and $D-\bar{D}$ mixing}

\subsubsection{Single scalar model}

For the $K_{L}-K_{S}$ mass difference and $D-\bar{D}$ mixing the Hamiltonian has the form

$$
\begin{aligned}
\mathcal{H}_{\mathrm{eff}}^{K_{L} K_{S}} & =C_{K_{L} K_{S}}\left(\bar{s}_{\alpha} \gamma^{\mu} P_{L} d_{\alpha}\right)\left(\bar{s}_{\beta} \gamma^{\mu} P_{L} d_{\beta}\right), \\
\mathcal{H}_{\mathrm{eff}}^{D \bar{D}} & =C_{D \bar{D}}\left(\bar{c}_{\alpha} \gamma^{\mu} P_{L} u_{\alpha}\right)\left(\bar{c}_{\beta} \gamma^{\mu} P_{L} u_{\beta}\right)
\end{aligned}
$$

where the $2 \sigma$ bound is given by

$$
\begin{aligned}
C_{K_{L} K_{S}} & =9 \times 10^{-7} \mathrm{TeV}^{-2} \\
C_{D D} & =2.7 \times 10^{-7} \mathrm{TeV}^{-2}
\end{aligned}
$$

The calculation of the box diagrams are the same as for $B_{s}-\bar{B}_{s}$ mixing with the dominant term coming from the quark mixing of the box involving only $Q_{3}$ current quarks. As a result the coefficients $C_{K_{L} K_{S}}\left(C_{D \bar{D}}\right)=\frac{\Gamma_{K_{L} K_{S}}\left(\Gamma_{D \bar{D}}\right)}{128 \pi^{2} m_{\Psi_{Q}}^{2}}$ for the single scalar model are given by

$$
\begin{aligned}
\Gamma_{K_{L} K_{S}} & \approx\left(V_{3 d}^{D *} V_{3 s}^{D}\right)^{2}\left|\Gamma_{b}\right|^{4} \\
\Gamma_{D \bar{D}} & \approx\left(V_{3 u}^{U *} V_{3 c}^{U}\right)^{2}\left|\Gamma_{b}\right|^{4}
\end{aligned}
$$

Due to the small mixing angles this is so heavily suppressed that the $K_{L}-K_{S}$ mass difference and $D-\bar{D}$ mixing do not provide a bound on the coupling $\Gamma_{b}$. 


\subsubsection{Two scalar model}

The situation is quite different for the two scalar model. If the down quark mixing dominates the $K_{L}-K_{S}$ mass difference gives the most stringent bound on the coupling $\widetilde{\Gamma}_{s}$ of $\tilde{\Phi}$ to the second current quark family because the mixing angle to the first family is large with $V_{2 d}^{D} \approx V_{c d}^{\text {CKM }}$. Thus in the two scalar model lepton universality violation effects are expected to be larger than in the single scalar case.

In the benchmark model, $m_{\Psi_{\ell}}=m_{\Phi_{L}}=100 \mathrm{GeV}, m_{\Phi_{H}}=400 \mathrm{GeV}$ and $m_{\Psi_{Q}}=$ $2.0 \mathrm{TeV}$, with $V_{2 s}^{D} \approx 1.0, V_{3 d}^{D} \approx 0, V_{2 d}^{D} \approx 0.23$ and $V_{3 s}^{D} \approx 0.042$, the $K_{L}-K_{S}$ mass difference is dominated by the $\left|\widetilde{\Gamma}_{s}\right|^{4}$ term and we find the bound $\left|\widetilde{\Gamma}_{s}\right|<0.57$. $^{12}$ For the $D-\bar{D}$ mixing case the bound is slightly stronger but can readily be satisfied with the same $\widetilde{\Gamma}_{s}$ if the up sector mixing angle is smaller, $V_{2 u}^{U}<0.5 V_{c d}^{\mathrm{CKM}}$. Note that these bounds may be evaded if there is a significant cancellation between the contributing box graphs (cf. section 4.1).

\section{$5.2 \quad B_{s}-\bar{B}_{s}$ mixing}

For ease of comparison we will use the $2 \sigma$ bounds quoted in [20]

$$
C_{B \bar{B}}\left(\mu_{H}\right) \in[-2.1,0.6] \times 10^{-5} \mathrm{TeV}^{-2} \quad(\text { at } 2 \sigma),
$$

\subsubsection{Single scalar model}

For the simple single scalar model introduced in section 2 the bound for the degenerate mass case, $x_{Q}=x_{\ell}=1$, is given by

$$
\left|\Gamma_{b}^{m} \Gamma_{s}^{m}\right| \approx\left|V_{3 b} V_{3 s}^{*}\right|\left|\Gamma_{b}\right|^{2} \leq 0.30 \frac{m_{\Psi}}{2 \mathrm{TeV}} .
$$

which gives

$$
\left|\Gamma_{b}\right| \leq 2.6 \sqrt{\frac{m_{\Psi}}{2 \mathrm{TeV}}}
$$

To illustrate the sensitivity to the mass spectrum, we will consider the extreme case, $x_{Q} \approx 400, x_{\ell} \approx 1$ in which the scalar and vector-like lepton are as light as possible, of order $100 \mathrm{GeV}$, consistent with present bounds, while the vector-like quark is of order $2 \mathrm{TeV}$ to be consistent with LHC bounds. In this case the bound is strengthened slightly giving

$$
\left|\Gamma_{b}^{m} \Gamma_{s}^{m}\right| \leq 0.18 \frac{m_{\Psi}}{2 \mathrm{TeV}} .
$$

which gives

$$
\left|\Gamma_{b}\right| \leq 2.0 \sqrt{\frac{m_{\Psi}}{2 \mathrm{TeV}}}
$$

The left panel in figure 3 shows this upper bound as a function of $x_{Q}$. The bound is given at $m_{\Psi_{Q}}=2 \mathrm{TeV}$, but other values are obtained by the indicated scaling, $m_{\Psi_{Q}} / 2 \mathrm{TeV}$.

\footnotetext{
${ }^{12}$ Note that suppressing the $V_{2 d}^{D}$ contribution to the Cabibbo angle is not viable because of the complementarity of the bounds from $K-\bar{K}$ and $D-\bar{D}$ mixing.
} 

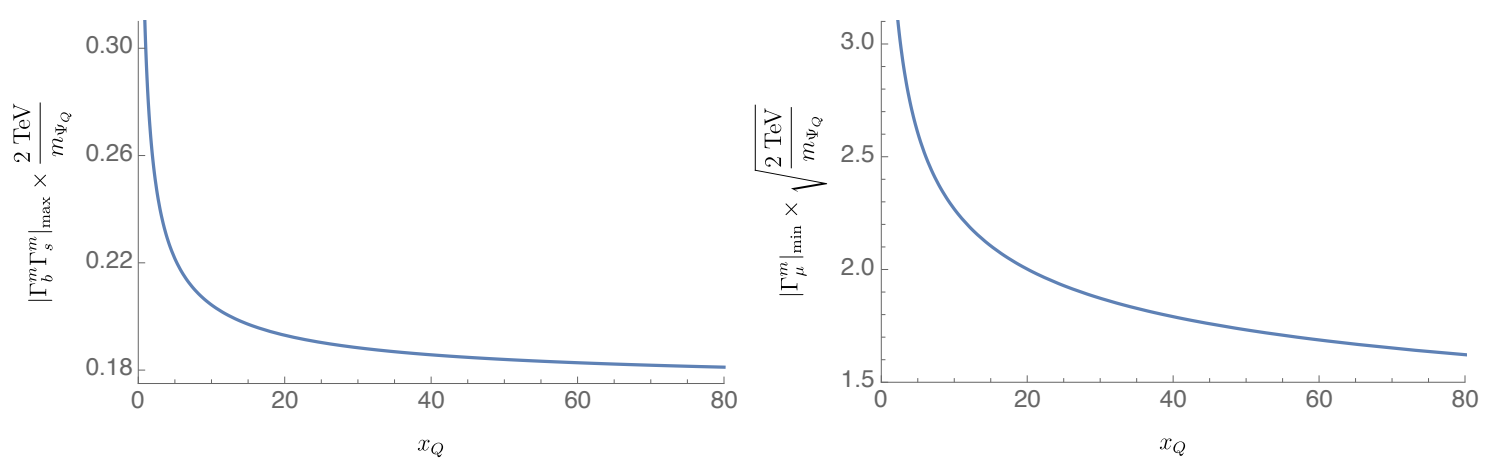

Figure 3. Comparison of the upper bound, for the simple single scalar model introduced in section 2 , on $\left|\Gamma_{b}^{m} \Gamma_{s}^{m}\right|$ from $B_{s}-\bar{B}_{s}$ mixing (left panel) with the lower bound on $\left|\Gamma_{\mu}^{m}\right|$ from $b \rightarrow s \mu \mu$ for $x_{\ell}=1$ (right panel), as a function of $x_{Q}$.

\subsubsection{Two scalar model}

As discussed above the suppression of the $B-\bar{B}$ amplitude requires that the scalar mass difference, $\delta m$ be small relative to $m_{\Psi_{Q}}$ as in our benchmark model. The bound on $\Gamma_{b}$ depends on the mixing angle $V_{3 s}^{D}$ and, for the extreme choices of this angle our benchmark model gives:

Down quark mixing vanishes, $\boldsymbol{V}_{\mathbf{3} s}^{\boldsymbol{D}}=\mathbf{0}$. Using the benchmark point $m_{\ell}=m_{L}=$ $100 \mathrm{GeV}, m_{H}=400 \mathrm{GeV}$ and $m_{\Psi}=2 \mathrm{TeV}$ we find

$$
C_{B \bar{B}} \approx 0.022 \frac{\left(\Gamma_{b}^{*} \widetilde{\Gamma}_{s}\right)^{2}}{512 \pi^{2} m_{\Psi_{Q}}^{2}},
$$

Taking $\left|\widetilde{\Gamma}_{s}\right|=0.57$, as needed for suppressing the $K_{L}-K_{S}$ mass difference, this gives, at the $2 \sigma$ level, $\left|\Gamma_{b}\right|<4.1$.

Down quark mixing maximal, $\boldsymbol{V}_{\mathbf{3} \boldsymbol{s}}^{\boldsymbol{D}}=\boldsymbol{V}_{\boldsymbol{c b}}^{\mathrm{CKM}}$. The left panel of figure 4 plots the $2 \sigma$-allowed region (shaded) in the $\widetilde{\Gamma}_{s}$ vs $\Gamma_{b}$ plane, for $m_{\Phi_{L}}=100 \mathrm{GeV}, m_{\Phi_{H}}=400 \mathrm{GeV}$, and $m_{\Psi_{Q}}=2 \mathrm{TeV}$, and $V_{3 s}^{D *}=-V_{2 b}^{D}=0.042$. Taking $\left|\widetilde{\Gamma}_{s}\right|=0.57$, we find the bound is satisfied at the $2 \sigma$ level by $\left|\Gamma_{b}\right|<1.8$

Finally, to demonstrate the suppression discussed in section 4.4.1 when $\delta \Gamma \rightarrow 0$, the right panel of figure 4 shows that the bounds disappear in this limit. The region shown is for the equal mass limit at $m_{\Phi_{L}}=m_{\Phi_{H}}=200 \mathrm{GeV}$, with $m_{\Psi_{Q}}=2 \mathrm{TeV}$ and $V_{3 s}^{D *}=$ $-V_{2 b}^{D}=0.042$. This limit is viable if there is a strong cancellation in $K^{0}-\bar{K}^{0}$ and $D-\bar{D}$ mixing.

\section{$5.3 \quad b \rightarrow s \mu \mu$}

We use the $2 \sigma$ bound quoted in [20]

$$
-1.28 \mathrm{TeV}^{-2} \leq C_{9}=-C_{10} \leq-0.49 \mathrm{TeV}^{-2} \quad(\text { at } 2 \sigma) .
$$



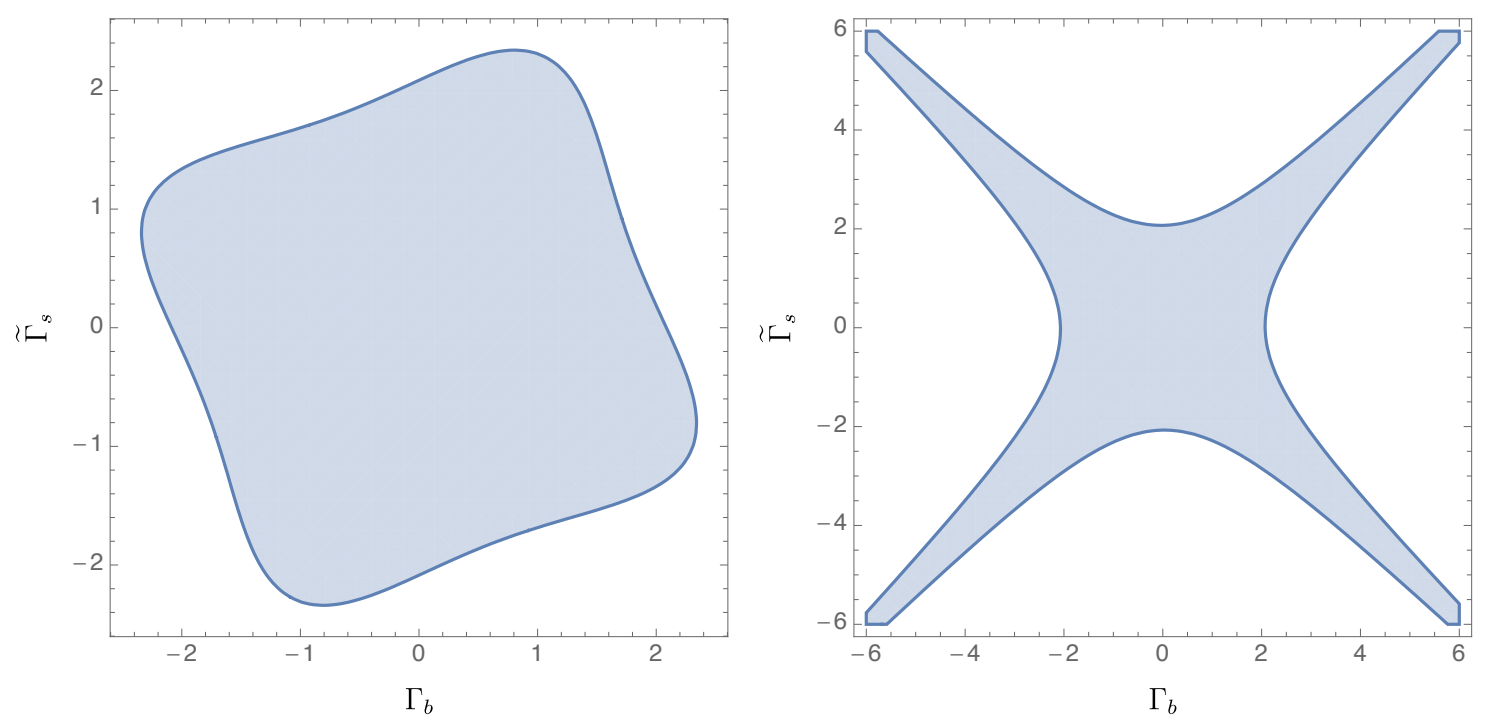

Figure 4. Allowed region (shaded) in the $\widetilde{\Gamma}_{s}$ vs $\Gamma_{b}$ plane at $2 \sigma$ in the two scalar model from $B_{s}-\bar{B}_{s}$ mixing. Left panel: using eq. (4.7), taking $m_{\Phi_{L}}=100 \mathrm{GeV}, m_{\Phi_{H}}=400 \mathrm{GeV}$, and $m_{\Psi_{Q}}=2 \mathrm{TeV}$, and $V_{3 s}^{D *}=-V_{2 b}^{D}=0.042$. Right panel: the equal mass limit at $m_{\Phi_{L}}=m_{\Phi_{H}}=200 \mathrm{GeV}$, with $m_{\Psi_{Q}}=2 \mathrm{TeV}$ and $V_{3 s}^{D *}=-V_{2 b}^{D}=0.042$; the absence of a bound in the simultaneous $\delta m \rightarrow 0$ and $\delta \Gamma \rightarrow 0$ limits is evident.

\subsubsection{Single scalar model}

In the degenerate mass case the function $F\left(x_{Q}, x_{\ell}\right)=1 / 3$ in eq. (2.3) and, for $m_{\Phi}=$ $2 \mathrm{TeV}$, the coupling is uncomfortably large, close to the nonperturbative limit, $\left|\Gamma_{\mu}\right| \geq 3.8$. However the bound is significantly weakened in the extreme case $x_{Q} \approx 400, x_{\ell} \approx 1$ with $F\left(x_{Q}, x_{\ell}\right)=1.1 \times 10^{-2}$ giving $1.3 \leq\left|\Gamma_{\mu}\right| \leq 2.2$, for the largest possible value of $\left|\Gamma_{b}^{m} \Gamma_{s}^{m}\right|$ given in eq. (5.10) or equivalently for $\left|\Gamma_{b}\right|=2$ as given in eq. (5.11). To facilitate comparison between models we also quote the result for the benchmark choice, $\Gamma_{b}=1.5$, which gives the bound $2.2>\left|\Gamma_{\mu}\right| \geq 1.8$.

\subsubsection{Two scalar model}

Down quark mixing vanishes, $\boldsymbol{V}_{\mathbf{3} \boldsymbol{s}}^{\boldsymbol{D}}=\mathbf{0}$. For the same benchmark point used in obtaining eq. (5.12), this pure scalar mixing case gives

$$
C_{9}^{\text {box }}=-C_{10}^{\text {box }} \approx-\frac{2.1 \Gamma_{b}^{*} \widetilde{\Gamma}_{s}\left|\Gamma_{\mu}\right|^{2}}{128 \pi \alpha_{E M} m_{\Psi_{Q}}^{2}}
$$

For the benchmark choice $\left|\Gamma_{b}\right|=1.5,\left|\widetilde{\Gamma}_{s}\right|=0.57$ the bound on $\Gamma_{\mu}$ is given by $2.9>\left|\Gamma_{\mu}\right| \geq 1.8$.

Down quark mixing maximal, $\boldsymbol{V}_{\mathbf{3} s}^{D}=\boldsymbol{V}_{\boldsymbol{c b}}^{\mathrm{CKM}}$. For the benchmark point we find $2.2>\left|\Gamma_{\mu}\right| \geq 1.4$. 


\subsection{Anomalous magnetic moment of the muon}

The effective Hamiltonian has the form

$$
\mathcal{H}_{\mathrm{eff}}^{a_{\mu}}=-a_{\mu} \frac{e}{4 m_{\mu}}\left(\bar{\mu} \sigma^{\lambda \nu} \mu\right) F_{\lambda \nu}
$$

where $a_{\mu} \equiv(g-2)_{\mu} / 2$ is the anomalous magnetic moment of the muon.

\subsubsection{Single scalar model}

The one loop graph, figure 4 of [20], gives a contribution to it of the form

$$
\Delta a_{\mu}=\frac{m_{\mu}^{2}\left|\Gamma_{\mu}\right|^{2}}{8 \pi^{2}} \frac{F_{7}\left(x_{\ell}\right)}{m_{\Phi}^{2}},
$$

where

$$
F_{7}(x)=\frac{x^{3}-6 x^{2}+6 x \log x+3 x+2}{12(x-1)^{4}} .
$$

In the limit of equal masses

$$
\Delta a_{\mu}=5.9 \times 10^{-12}\left|\Gamma_{\mu}\right|^{2}\left(\frac{1 \mathrm{TeV}}{m_{\Phi}}\right) .
$$

negligible when compared to the discrepancy of the Standard Model prediction from the experimental measurement

$$
\Delta a_{\mu}=a_{\mu}^{\exp }-a_{\mu}^{\mathrm{SM}}=(2.36 \pm 0.87) \times 10^{-9} .
$$

Clearly $\Delta a_{\mu}$ increases significantly in the non-degenerate case with light $\Psi_{\ell}$ and $\Phi$. We will consider the benchmark point $m_{\Psi_{\ell}}=105 \mathrm{GeV}$ and $m_{\Phi}=62 \mathrm{GeV} .{ }^{13}$ To reduce the discrepancy of $a_{\mu}$ from $2.7 \sigma$ to $1 \sigma$ requires $\Gamma_{\mu}=1.4$ while to remove the discrepancy completely requires $\Gamma_{\mu}=1.8$. It is noticeable that these values are not far from the bounds derived above.

\subsubsection{Two scalar model}

The anomalous magnetic moment does not involve flavour mixing so one gets a unique prediction. In this case

$$
\Delta a_{\mu}=\frac{m_{\mu}^{2}\left|\Gamma_{\mu}\right|^{2}}{16 \pi^{2}}\left(\frac{F_{7}\left(x_{\Phi_{L}}\right)}{m_{\Phi_{L}}^{2}}+\frac{F_{7}\left(x_{\Phi_{H}}\right)}{m_{\Phi_{H}}^{2}}\right),
$$

where $x_{\Phi_{L, H}}=m_{\Psi_{\ell}}^{2} / m_{\Phi_{L, H}}^{2}$. For the benchmark point $m_{\Psi_{\ell}}=105 \mathrm{GeV}, m_{\Phi_{L}}=62 \mathrm{GeV}$, $m_{\Phi_{H}}=400 \mathrm{GeV}$, to reduce the discrepancy to $1 \sigma$ requires $\Gamma_{\mu}=1.9$ and to eliminate the discrepancy completely requires $\Gamma_{\mu}=2.4$. The contribution is sensitive to the mixing angle, $\theta$, associated with the scalar mass eigenstates and reducing this from the maximal mixing value, $\theta=\pi / 4$, to $\theta=\pi / 8$ the value needed change to 1.5 and 1.9 respectively. 


\begin{tabular}{|c|c|c|c|}
\hline \multirow{2}{*}{} & \multirow{2}{*}{ Single scalar } & \multicolumn{2}{|c|}{ Two scalar } \\
\cline { 3 - 4 } & & $V_{2 b}^{D}=0$ & $V_{2 b}^{D}=-0.043$ \\
\hline$B_{s}-\bar{B}_{s}$ & $\Gamma_{b}<2.0$ & $\Gamma_{b}<4.1(5.8)$ & $\Gamma_{b}<2.3(2.3)$ \\
\hline$b \rightarrow s \mu \mu$ & $3>\Gamma_{\mu}>1.8$ & $2.9(3.0)>\Gamma_{\mu}>1.8(1.9)$ & $2.2(2.2)>\Gamma_{\mu}>1.4(1.3)$ \\
\hline$(g-2)_{\mu}$ & $\Gamma_{\mu}=1.4$ & $\Gamma_{\mu}=1.9(1.5)$ & $\Gamma_{\mu}=1.9(1.5)$ \\
\hline
\end{tabular}

Table 4. Comparison of the parameters needed for different models. In all cases we used $V_{2 s}^{D} \approx 1.0, V_{3 d}^{D} \approx 0, V_{2 d}^{D} \approx 0.23$. For the single scalar model we have used $m_{\Psi_{\ell}}=m_{\Phi}=100 \mathrm{GeV}$, and $m_{\Psi_{Q}}=2.0 \mathrm{TeV}$. For both two-scalar models we used $m_{\Psi_{\ell}}=m_{\Phi_{L}}=100 \mathrm{GeV}, m_{\Phi_{H}}=400 \mathrm{GeV}$ and $m_{\Psi_{Q}}=2.0 \mathrm{TeV}$, and $\left|\widetilde{\Gamma}_{s}\right|=0.57$, that satisfies the bounds from $K_{L}-K_{S}$ mass difference. In the two-scalar models, $b \rightarrow s \mu \mu$ is computed at the benchmark point $\left|\Gamma_{b}\right|=1.5$. In the last row the parameter values given reduce the discrepancy in $(g-2)_{\mu}$ from $2.7 \sigma$ to $1 \sigma$; for the single scalar model we have used $m_{\Psi_{\ell}}=105 \mathrm{GeV}$ and $m_{\Phi}=62 \mathrm{GeV}$, while for the two scalar models we used $m_{\Psi_{\ell}}=105 \mathrm{GeV}, m_{\Phi_{L}}=62 \mathrm{GeV}, m_{\Phi_{H}}=400 \mathrm{GeV}$. The terms in parenthesis correspond to non-maximal mixing with $\theta=\pi / 8$ in eq. (4.2).

\subsection{Comparison}

In table 4 we compare the results obtained above for the benchmark points. The first of the two columns under "Two scalar", $V_{2 b}^{D}=0$, corresponds to the pure scalar induced mixing case. For the two scalar model we assume the bound from $K_{L}-K_{S}$ mass difference, $\left|\widetilde{\Gamma}_{s}\right| \leq 0.57$ is saturated, and we show, in parenthesis, values corresponding to non-maximal scalar mixing with $\theta=\pi / 8$. In all cases we used $V_{2 s}^{D} \approx 1.0, V_{3 d}^{D} \approx 0, V_{2 d}^{D} \approx 0.23$. For the single scalar model we have used $m_{\Psi_{\ell}}=m_{\Phi}=100 \mathrm{GeV}$, and $m_{\Psi_{Q}}=2.0 \mathrm{TeV}$, while for the two-scalar models we used $m_{\Psi_{\ell}}=m_{\Phi_{L}}=100 \mathrm{GeV}, m_{\Phi_{H}}=400 \mathrm{GeV}$ and $m_{\Psi_{Q}}=2.0 \mathrm{TeV}$. In the two-scalar models, $b \rightarrow s \mu \mu$ is computed at the benchmark point $\left|\Gamma_{b}\right|=1.5$. For $\Delta a_{\mu}$ the parameter values given reduce the discrepancy in $(g-2)_{\mu}$ from $2.7 \sigma$ to $1 \sigma$, and, as explained in sections 5.4 and 6 , for the single scalar model we have used a different benchmark point, namely, $m_{\Psi_{\ell}}=105 \mathrm{GeV}$ and $m_{\Phi}=62 \mathrm{GeV}$, while for the two scalar models we used $m_{\Psi_{\ell}}=105 \mathrm{GeV}, m_{\Phi_{L}}=62 \mathrm{GeV}, m_{\Phi_{H}}=400 \mathrm{GeV}$.

\section{$5.6 \mu \rightarrow e \gamma$}

Following [20] the decay $\mu \rightarrow e \gamma$ is described by the effective Hamiltonian

$$
\mathcal{H}_{\mathrm{eff}}^{\mu \rightarrow e \gamma}=-C_{\mu \rightarrow e \gamma} m_{\mu}\left(\bar{e} \sigma^{\mu \nu} P_{R} \mu\right) F_{\mu \nu}
$$

from which the branching ratio is obtained according to

$$
\operatorname{Br}(\mu \rightarrow e \gamma)=\frac{m_{\mu}^{5}}{4 \pi} \tau_{\mu}\left|C_{\mu \rightarrow e \gamma}\right|^{2},
$$

where $\tau_{\mu}$ denotes the life-time of the muon. The experimental upper limit $[43,44]$ is currently given by

$$
\operatorname{Br}^{\exp }(\mu \rightarrow e \gamma) \leq 4.2 \times 10^{-13}, \quad(90 \% \text { C.L. }),
$$

\footnotetext{
${ }^{13}$ This is taken because, even in the case $\Phi$ is stable, this is an experimentally allowed point, cf. section 6 .
} 
giving the bound

$$
m_{\mu}^{2}\left|C_{\mu \rightarrow e \gamma}\right|<3.9 \times 10^{-15} .
$$

The coefficient $C_{\mu \rightarrow e \gamma}$ is related to the scalar contribution to the anomalous magnetic moment of the muon,

$$
C_{\mu \rightarrow e \gamma} \approx \frac{e}{m_{\mu}^{2}} V_{2 e}^{E} \Delta a_{\mu}
$$

where $V_{2 e}^{E}$ is the component of the electron in the muon current eigenstate. Large enough $\Delta a_{\mu}$ to explain the discrepancy of the SM prediction with experiment requires $V_{2 e}^{E}<10^{-5}$. Its value is model dependent. In the model of section $3, V_{2 e}^{E} \propto\left(\chi / M_{q}\right)^{4}$. Given the uncertainty in the string of couplings and messenger masses generating this term such a suppression may be sufficient to satisfy current experimental bounds. In the model presented in appendix A the mixing vanishes.

\section{$5.7 \quad Z \rightarrow \mu^{+} \mu^{-}$}

In the two scalar model the $Z$ penguin contribution involving $\Psi_{\ell}$ and $\Phi$ to the correction to the coupling of the left handed muon to the $Z$-boson is given by [20]

$$
\frac{\delta g_{L \mu}}{g_{L \mu}^{\mathrm{SM}}}\left(q^{2}\right)=-\frac{1}{64 \pi^{2}} \frac{q^{2}}{m_{\Psi_{\ell}}^{2}}\left|\Gamma_{\mu}\right|^{2}\left(\widetilde{G}_{9}\left(x_{\Phi_{L}}\right)+\widetilde{G}_{9}\left(x_{\Phi_{H}}\right)\right)
$$

where

$$
G_{9}(x)=\frac{7-36 x+45 x^{2}-16 x^{3}+6(2 x-3) x^{2} \log x}{36(x-1)^{4}}, \quad \widetilde{G}_{9}(x)=x^{-1} G_{9}\left(x^{-1}\right) .
$$

The LEP measurement [48] $g_{L \mu}^{\exp }\left(m_{Z}^{2}\right)=-0.2689 \pm 0.0011$ implies

$$
\left|\frac{\delta g_{L \mu}}{g_{L \mu}^{\mathrm{SM}}}\left(m_{Z}^{2}\right)\right| \leq 0.8 \% \quad(2 \sigma) .
$$

For the case $m_{\Psi_{\ell}}=100 \mathrm{GeV}, m_{\Phi_{L}}=80 \mathrm{GeV}, m_{\Phi_{H}}=400 \mathrm{GeV}$, we find $\frac{\delta g_{L \mu}}{g_{L \mu}^{\mathrm{SM}}}\left(M_{Z}^{2}\right)=$ $9 \times 10^{-4}\left|\Gamma_{\mu}\right|^{2}$, well within the LEP bound for perturbative couplings. For the single scalar case the new contribution is smaller.

\section{Bounds on the new particle masses and their dark matter abundance}

In the Abelian family symmetry models of section 3.2 only the heavy vector-like quark and lepton states and the scalar states $\Phi$ and $\tilde{\Phi}$ are odd under the $Z_{2}$ symmetry. As a result the lightest $Z_{2}$ odd state will be stable and a candidate for dark matter. In the mass range of interest here direct searches exclude the possibility that the DM candidate is the heavy lepton so one is left with the possibility that the lightest scalar is the DM.

In this case the bounds on the heavy quark mass come from pair production of the coloured vector-like quarks and their subsequent decay into the light scalar plus a quark $\left(p p \rightarrow \Psi_{Q} \bar{\Psi}_{Q} ; \Psi_{Q} \rightarrow q \Phi_{L}\right)$ where $q$ is dominantly a third generation quark. The LHC 
limits for this case have been studied in detail by Kawamura et al. [50] ${ }^{14}$ and their results are shown in their figure 1, case A. One sees that for a scalar mass of $O(100) \mathrm{GeV}$ the heavy quark should be heavier than $1.6 \mathrm{TeV}$.

For the model with just a single scalar, $\Phi$, the possibility it is dark matter has been studied in detail by Calibbi et al. [52]. The model corresponds to their LL1 model and in figure 4 they show the dark matter abundance in the $m_{S}\left(\equiv m_{\Phi}\right), m_{L}\left(\equiv m_{\Psi_{\ell}}\right)$ plane for a range of Yukawa couplings, $\lambda_{L}\left(\equiv \Gamma_{\mu}\right)$. In figure 6 they plot the dark matter abundance in the $\lambda_{L}, m_{S}$ plane for a couple of values for $m_{L}$. Also shown are the LHC limits on $m_{S}$ and the $1 \sigma$ and $2 \sigma$ limits on $(g-2)_{\mu}$.

One may see that there is a small window with $m_{\Phi}=(60-80) \mathrm{GeV}$ which has not been excluded by the LHC. Interestingly it is possible that $\Phi$ makes up all of dark matter in this mass range, depending on the heavy lepton mass and its coupling to the scalar. For example this is possible for $m_{\Psi_{\ell}}=105 \mathrm{GeV}$, a scalar mass $m_{\Phi_{L}}=62 \mathrm{GeV}$ and $\Gamma_{\mu}=1.2$, a value a bit below the allowed range coming from $b \rightarrow s \mu \mu$. With this Yukawa coupling the discrepancy in the anomalous magnetic moment is reduced to $1.5 \sigma$.

For the two scalar model the window applies to the light scalar mass, $m_{\Phi_{L}}=(60-$ 80) $\mathrm{GeV}$. In this case, for maximal mixing, $\lambda_{L} \equiv \Gamma_{\mu} / \sqrt{2}$ so, for a heavy lepton mass of $105 \mathrm{GeV}$, the light scalar can make up all of dark matter for $\Gamma_{\mu}=1.7$. This is in the range required to explain $b \rightarrow s \mu \mu$ and within $1.3 \sigma$ of the anomalous magnetic moment. The latter is quite sensitive to the mass of the heavier scalar and the discrepancy falls to $1 \sigma$ for $m_{\Phi_{H}}=200 \mathrm{GeV}$.

Note that the bounds on the vector-like quarks and leptons and the new scalars are significantly weakened in the case discussed in appendix B when the lightest new state can decay rapidly into Standard Model states.

\section{Summary and conclusions}

In this paper we have explored the possibility that the neutral current anomalies observed in B decays come from loop effects generated by Yukawa couplings of SM fermions to new heavy SM singlet scalars and new heavy vector-like quark and lepton SM doublets. We have shown that the phenomenologically required structure of the Yukawa couplings can be ensured by a simple Abelian family symmetry and that the same symmetry can generate and acceptable pattern of fermion masses and mixing angles, thus relating the lepton non-universality observed in the B-sector to the non-universality of the fermion mass matrix.

We have analysed two simple models, one with a single heavy scalar in which flavour changing is driven solely by the mixing in the fermion sector and a second involving two heavy scalars in which flavour changing is generated by fermion mixing and/or scalar mixing. In both cases, for the vector-like quark much heavier than the other new states, it is possible to generate the effective operators, $O_{9}$ and $O_{10}$ with coefficient large enough to explain the B-anomaly without violating the stringent bound coming from $B_{s}-\bar{B}_{s}$ mixing.

\footnotetext{
${ }^{14}$ See also [49].
} 
Indeed in the two scalar model it is possible to avoid this bound completely with the caveat that one also has a strong cancellation in $K-\bar{K}$ and $D-\bar{D}$ mixing. We have also checked that the Yukawa couplings needed do not generate an unacceptable contribution to the $K_{L}-K_{S}$ mass difference and $D-\bar{D}$ mixing or to other lepton family number or universality violating processes. While the Yukawa couplings required are quite large, they remain in the perturbative regime with the further new states needed to avoid their Landau poles only required above a scale of $O\left(10^{6}\right) \mathrm{GeV}$. It is interesting to note that the same coupling generates a contribution to the muon anomalous magnetic moment that resolves the observed discrepancy of the experimental value with the SM prediction. For this to be the case the lightest scalar and vector-like lepton should be quite light, of $O(100) \mathrm{GeV}$, but if they decay rapidly to SM states there will be no significant missing energy signal and the best limit is the LEP limit for the heavy lepton at $100 \mathrm{GeV}$. The scalar cannot be directly pair produced and so the limit on its mass is much milder.

On the other hand a simple $Z_{2}$ extension of the family symmetry ensures that the new states are distinguished from the SM states so that they can only be produced in pairs and the lightest state, which must be a scalar, is stable and a DM candidate. In the case of the two scalar model the DM abundance for the Yukawa coupling needed to explain the $\mathrm{B}$-anomalies is at the level that it can make up all of DM for a scalar mass of $O(100) \mathrm{GeV}$. Of course it is necessary to check that the new heavy states should not have been observed to date and in this case there are significant missing energy signals. The bounds on the coloured quarks are quite severe, requiring them to be above $1.5 \mathrm{TeV}$ and accordingly our numerical estimates were done for a $2 \mathrm{TeV}$ vector-like doublet of quarks. The other states must be much lighter and it turns out that there is a viable window with the lightest scalar mass of $O(60-80) \mathrm{GeV}$ and the heavy lepton mass of $O(100) \mathrm{GeV}$. In this region it is possible simultaneously to explain the neutral B anomalies, the muon anomalous magnetic moment and the DM abundance. It should be possible for the LHC experimental searches to fully explore this region in the near future.

\section{Acknowledgments}

We are grateful to Alan Barr, Andrzej Buras, Yosi Nir, Gilad Perez, Andreas Weiler, Robert Ziegler and especially Andreas Crivellin and Kazuki Sakurai for helpful discussions and to the Theory Group, CERN, where much of the work was done. The work of SP is partially supported by the National Science Centre, Poland, under research grants DEC2015/18/M/ST2/00054, DEC-2015/19/B/ST2/02848 and DEC-2016/23/G/ST2/04301. The work of BG is partially supported by the Department of Energy, U.S.A., under research grant DE-SC0009919.

\section{A Elimination of lepton flavour changing processes}

While the lepton family mixing is small enough to avoid violating bounds on lepton flavour violating processes, $\tau \rightarrow \mu \gamma, \mu \rightarrow e \gamma$ etc. the expectation in this model is that the branching ratios are close to these bounds. Thus it is of interest to check whether these can be further 
suppressed. Indeed this is relatively simple through the introduction of a further family symmetry. A simple example is to add a $Z_{3}$ discrete symmetry under which the 3 different lepton families transform as $1, \alpha$ and $\alpha^{2}$ where $\alpha$ is the cube root of unity. In this case there can be no lepton family mixing and the current eigenstates are the mass eigenstates. If, in addition, $\Psi_{\ell,(L, R)}$ transforms in the same way as the muon under $Z_{3}$ then the box graph will only involve muons and generate $b \rightarrow s \mu \mu$ as desired. Because the lepton families do not mix it is possible to simplify their charge structure under $\mathrm{U}(1)_{F}$. The choice given in eq. (A.1) gives an acceptable lepton mass hierarchy with $\frac{m_{\mu}}{m_{\tau}}=O(\epsilon)$ and $\frac{m_{e}}{m_{\mu}}=O\left(\epsilon^{3}\right)$.

\begin{tabular}{c|cccccccccccccccccc} 
& $Q_{1}$ & $q_{1}$ & $Q_{2}$ & $q_{2}$ & $Q_{3}$ & $q_{3}$ & $L_{1}$ & $l_{1}$ & $L_{2}$ & $l_{2}$ & $L_{3}$ & $l_{3}$ & $\Psi_{Q,(L, R)}$ & $\Psi_{\ell,(L, R)}$ & $\Phi$ & $\tilde{\Phi}$ & $H$ & $\chi$ \\
\hline$Q_{F}$ & 3 & -1 & 2 & 0 & 0 & 0 & 2 & -2 & -1 & 0 & 0 & 0 & 2 & 1 & -2 & 0 & 0 & 1 \\
\hline$Z_{3}$ & $1(\alpha)$ & $1(\alpha)$ & 1 & 1 & 1 & 1 & 1 & 1 & $\alpha$ & $\alpha$ & $\alpha^{2}$ & $\alpha^{2}$ & 1 & $\alpha$ & 1 & 1 & 1 & 1
\end{tabular}

With these $Z_{3}$ charges given in eq. (A.1) there is no lepton family mixing. The muon mass remains of $O(\epsilon)$ relative to the $\tau$ mass but the electron mass is now $O\left(\epsilon^{3}\right)$ relative to the muon mass, still phenomenologically acceptable given the unknown $O(1)$ couplings involved.

The quark masses and mixings are unchanged compared to the model discussed in section 3 with couplings of $Q_{1}$ to the vector-like quark generating additional non-universal lepton decay modes. These can readily be forbidden if the first generation quark doublet and down quark transform non-trivially under $Z_{3}$ as shown by the terms in brackets in eq. (A.1). In this case the mixing to the first generation of quarks must occur in the up quark sector.

Neutrino massese are presumed to be generated at very short distances. The $Z_{3}$ symmetry only allows $L_{2} L_{3}$ terms in a Weinberg operator for see-saw neutrino masses. However, it is natural to expect that the dynamics responsible for breaking lepton number does not respect $Z_{3}$.

\section{B Simplified model without a dark matter candidate}

If one is not concerned to have the possibility that the new heavy states are dark matter candidates it is possible to simplify the original model by dropping the $Z_{2}$ symmetry. In this case there are additional terms allowed, for example

$$
\begin{aligned}
\mathcal{L}_{\text {eff }}^{M}= & M^{\prime} \bar{Q}_{2} P_{R} \Psi_{Q}+\bar{\Psi}_{Q} P_{R} q_{2} H\left(\frac{\chi}{M_{q}}\right)^{2}+\bar{\Psi}_{Q} P_{R} q_{3} H\left(\frac{\chi}{M_{q}}\right)^{2}+\bar{\Psi}_{Q} P_{R} q_{1} H\left(\frac{\chi}{M_{q}}\right)^{3} \\
& +\widetilde{\Gamma}_{s} \bar{\Psi}_{Q} P_{R} \Psi_{Q} \tilde{\Phi}
\end{aligned}
$$

The first four terms induce mixing between $q_{i}, Q_{2}$ and $\Psi_{Q}$; together with the corresponding mass terms in section 3 these induce $4 \times 4$ mass matrices for up and down type quarks so that, e.g., the unitary matrices that diagonalize these are $4 \times 4$. The last term is an interaction, and both this term and the mass mixing clearly allow the original $Z_{2}$ odd states to decay so they are no longer dark matter candidates. 
Open Access. This article is distributed under the terms of the Creative Commons Attribution License (CC-BY 4.0), which permits any use, distribution and reproduction in any medium, provided the original author(s) and source are credited.

\section{References}

[1] LHCb collaboration, Test of lepton universality using $B^{+} \rightarrow K^{+} \ell^{+} \ell^{-}$decays, Phys. Rev. Lett. 113 (2014) 151601 [arXiv: 1406.6482] [INSPIRE].

[2] LHCb collaboration, Test of lepton universality with $B^{0} \rightarrow K^{* 0} \ell^{+} \ell^{-}$decays, JHEP 08 (2017) 055 [arXiv: 1705.05802] [INSPIRE].

[3] LHCb collaboration, Measurement of Form-Factor-Independent Observables in the Decay $B^{0} \rightarrow K^{* 0} \mu^{+} \mu^{-}$, Phys. Rev. Lett. 111 (2013) 191801 [arXiv:1308.1707] [InSPIRE].

[4] LHCb collaboration, Angular analysis of the $B^{0} \rightarrow K^{* 0} \mu^{+} \mu^{-}$decay using $3 \mathrm{fb}^{-1}$ of integrated luminosity, JHEP 02 (2016) 104 [arXiv:1512.04442] [INSPIRE].

[5] Belle collaboration, S. Wehle et al., Lepton-Flavor-Dependent Angular Analysis of $B \rightarrow K^{*} \ell^{+} \ell^{-}$, Phys. Rev. Lett. 118 (2017) 111801 [arXiv:1612.05014] [inSPIRE].

[6] LHCb collaboration, Differential branching fractions and isospin asymmetries of $B \rightarrow K^{(*)} \mu^{+} \mu^{-}$decays, JHEP 06 (2014) 133 [arXiv:1403.8044] [INSPIRE].

[7] LHCb collaboration, Angular analysis and differential branching fraction of the decay $B_{s}^{0} \rightarrow \phi \mu^{+} \mu^{-}$, JHEP 09 (2015) 179 [arXiv:1506.08777] [INSPIRE].

[8] W. Altmannshofer, P. Stangl and D.M. Straub, Interpreting Hints for Lepton Flavor Universality Violation, Phys. Rev. D 96 (2017) 055008 [arXiv: 1704.05435] [INSPIRE].

[9] G. D'Amico et al., Flavour anomalies after the $R_{K^{*}}$ measurement, JHEP 09 (2017) 010 [arXiv: 1704.05438] [INSPIRE].

[10] G. Hiller and I. Nisandzic, $R_{K}$ and $R_{K^{*}}$ beyond the standard model, Phys. Rev. D 96 (2017) 035003 [arXiv: 1704.05444] [INSPIRE].

[11] B. Capdevila, A. Crivellin, S. Descotes-Genon, J. Matias and J. Virto, Patterns of New Physics in $b \rightarrow s \ell^{+} \ell^{-}$transitions in the light of recent data, JHEP 01 (2018) 093 [arXiv: 1704.05340] [INSPIRE].

[12] L.-S. Geng, B. Grinstein, S. Jäger, J. Martin Camalich, X.-L. Ren and R.-X. Shi, Towards the discovery of new physics with lepton-universality ratios of $b \rightarrow$ sll decays, Phys. Rev. D 96 (2017) 093006 [arXiv: 1704.05446] [INSPIRE].

[13] M. Ciuchini et al., On Flavourful Easter eggs for New Physics hunger and Lepton Flavour Universality violation, Eur. Phys. J. C 77 (2017) 688 [arXiv:1704.05447] [INSPIRE].

[14] C.D. Froggatt and H.B. Nielsen, Hierarchy of Quark Masses, Cabibbo Angles and CP-violation, Nucl. Phys. B 147 (1979) 277 [INSPIRE].

[15] B. Gripaios, M. Nardecchia and S.A. Renner, Linear flavour violation and anomalies in $B$ physics, JHEP 06 (2016) 083 [arXiv: 1509.05020] [InSPIRE].

[16] J.M. Cline and J.M. Cornell, $R\left(K^{(*)}\right)$ from dark matter exchange, Phys. Lett. B 782 (2018) 232 [arXiv: 1711.10770] [INSPIRE].

[17] Z. Poh and S. Raby, Vectorlike leptons: Muon g-2 anomaly, lepton flavor violation, Higgs boson decays and lepton nonuniversality, Phys. Rev. D 96 (2017) 015032 [arXiv: 1705.07007] [INSPIRE]. 
[18] L. Dhargyal, A simple model to explain the observed muon sector anomalies, small neutrino masses, baryon-genesis and dark-matter, arXiv:1711.09772 [INSPIRE].

[19] L. Dhargyal and S.K. Rai, Implications of a vector-like lepton doublet and scalar Leptoquark on $R\left(D^{(*)}\right)$, arXiv: 1806.01178 [INSPIRE].

[20] P. Arnan, L. Hofer, F. Mescia and A. Crivellin, Loop effects of heavy new scalars and fermions in $b \rightarrow s \mu^{+} \mu^{-}$, JHEP 04 (2017) 043 [arXiv: 1608.07832] [INSPIRE].

[21] D. Das, C. Hati, G. Kumar and N. Mahajan, Scrutinizing R-parity violating interactions in light of $R_{K^{(*)}}$ data, Phys. Rev. D 96 (2017) 095033 [arXiv: 1705. 09188] [InSPIRE].

[22] BABAR collaboration, J.P. Lees et al., Evidence for an excess of $\bar{B} \rightarrow D^{(*)} \tau^{-} \bar{\nu}_{\tau}$ decays, Phys. Rev. Lett. 109 (2012) 101802 [arXiv:1205.5442] [INSPIRE].

[23] BaBAR collaboration, J.P. Lees et al., Measurement of an Excess of $\bar{B} \rightarrow D^{(*)} \tau^{-} \bar{\nu}_{\tau}$ Decays and Implications for Charged Higgs Bosons, Phys. Rev. D 88 (2013) 072012 [arXiv: 1303.0571] [INSPIRE].

[24] BeLLE collaboration, M. Huschle et al., Measurement of the branching ratio of $\bar{B} \rightarrow D^{(*)} \tau^{-} \bar{\nu}_{\tau}$ relative to $\bar{B} \rightarrow D^{(*)} \ell^{-} \bar{\nu}_{\ell}$ decays with hadronic tagging at Belle, Phys. Rev. $\mathbf{D}$ 92 (2015) 072014 [arXiv: 1507.03233] [INSPIRE].

[25] Belle collaboration, Y. Sato et al., Measurement of the branching ratio of $\bar{B}^{0} \rightarrow D^{*+} \tau^{-} \bar{\nu}_{\tau}$ relative to $\bar{B}^{0} \rightarrow D^{*+} \ell^{-} \bar{\nu}_{\ell}$ decays with a semileptonic tagging method, Phys. Rev. D 94 (2016) 072007 [arXiv: 1607. 07923] [INSPIRE].

[26] Belle collaboration, S. Hirose et al., Measurement of the $\tau$ lepton polarization and $R\left(D^{*}\right)$ in the decay $\bar{B} \rightarrow D^{*} \tau^{-} \bar{\nu}_{\tau}$, Phys. Rev. Lett. 118 (2017) 211801 [arXiv:1612.00529] [INSPIRE].

[27] BeLle collaboration, S. Hirose et al., Measurement of the $\tau$ lepton polarization and $R\left(D^{*}\right)$ in the decay $\bar{B} \rightarrow D^{*} \tau^{-} \bar{\nu}_{\tau}$ with one-prong hadronic $\tau$ decays at Belle, Phys. Rev. D 97 (2018) 012004 [arXiv: 1709.00129] [INSPIRE].

[28] LHCb collaboration, Measurement of the ratio of branching fractions $\mathcal{B}\left(\bar{B}^{0} \rightarrow D^{*+} \tau^{-} \bar{\nu}_{\tau}\right) / \mathcal{B}\left(\bar{B}^{0} \rightarrow D^{*+} \mu^{-} \bar{\nu}_{\mu}\right)$, Phys. Rev. Lett. 115 (2015) 111803 [Erratum ibid. 115 (2015) 159901] [arXiv: 1506.08614] [INSPIRE].

[29] LHCb collaboration, Measurement of the ratio of the $B^{0} \rightarrow D^{*-} \tau^{+} \nu_{\tau}$ and $B^{0} \rightarrow D^{*-} \mu^{+} \nu_{\mu}$ branching fractions using three-prong $\tau$-lepton decays, Phys. Rev. Lett. 120 (2018) 171802 [arXiv: 1708.08856] [INSPIRE].

[30] LHCb collaboration, Test of Lepton Flavor Universality by the measurement of the $B^{0} \rightarrow D^{*-} \tau^{+} \nu_{\tau}$ branching fraction using three-prong $\tau$ decays, Phys. Rev. D 97 (2018) 072013 [arXiv: 1711. 02505] [INSPIRE].

[31] LHCb collaboration, Measurement of the ratio of branching fractions $\mathcal{B}\left(B_{c}^{+} \rightarrow J / \psi \tau^{+} \nu_{\tau}\right) / \mathcal{B}\left(B_{c}^{+} \rightarrow J / \psi \mu^{+} \nu_{\mu}\right)$, Phys. Rev. Lett. 120 (2018) 121801 [arXiv: 1711.05623] [INSPIRE].

[32] R. Alonso, B. Grinstein and J. Martin Camalich, Lepton universality violation and lepton flavor conservation in B-meson decays, JHEP 10 (2015) 184 [arXiv:1505.05164] [INSPIRE].

[33] G. Hiller and M. Schmaltz, $R_{K}$ and future $b \rightarrow$ sll physics beyond the standard model opportunities, Phys. Rev. D 90 (2014) 054014 [arXiv: 1408.1627] [INSPIRE].

[34] R. Barbieri, G. Isidori, A. Pattori and F. Senia, Anomalies in B-decays and U(2) flavour symmetry, Eur. Phys. J. C 76 (2016) 67 [arXiv:1512.01560] [InSPIRE]. 
[35] N. Assad, B. Fornal and B. Grinstein, Baryon Number and Lepton Universality Violation in Leptoquark and Diquark Models, Phys. Lett. B 777 (2018) 324 [arXiv:1708.06350] [INSPIRE].

[36] L. Di Luzio, A. Greljo and M. Nardecchia, Gauge leptoquark as the origin of B-physics anomalies, Phys. Rev. D 96 (2017) 115011 [arXiv:1708.08450] [INSPIRE].

[37] L. Calibbi, A. Crivellin and T. Li, A model of vector leptoquarks in view of the B-physics anomalies, arXiv:1709.00692 [INSPIRE].

[38] R. Barbieri and A. Tesi, B-decay anomalies in Pati-Salam SU(4), Eur. Phys. J. C 78 (2018) 193 [arXiv: 1712.06844] [INSPIRE].

[39] M. Blanke and A. Crivellin, B Meson Anomalies in a Pati-Salam Model within the Randall-Sundrum Background, Phys. Rev. Lett. 121 (2018) 011801 [arXiv:1801.07256] [INSPIRE].

[40] A. Crivellin, C. Greub, F. Saturnino and D. Müller, Importance of Loop Effects in Explaining the Accumulated Evidence for New Physics in B Decays with a Vector Leptoquark, arXiv: 1807.02068 [INSPIRE].

[41] A. Greljo, D.J. Robinson, B. Shakya and J. Zupan, $R\left(D^{(*)}\right)$ from $W^{\prime}$ and right-handed neutrinos, JHEP 09 (2018) 169 [arXiv:1804.04642] [INSPIRE].

[42] HFLAV collaboration, Y. Amhis et al., Averages of b-hadron, c-hadron and $\tau$-lepton properties as of summer 2016, Eur. Phys. J. C 77 (2017) 895 [arXiv:1612.07233] [INSPIRE].

[43] MEG collaboration, J. Adam et al., New constraint on the existence of the $\mu^{+} \rightarrow e^{+} \gamma$ decay, Phys. Rev. Lett. 110 (2013) 201801 [arXiv:1303.0754] [InSPIRE].

[44] MEG collaboration, F. Cei et al., Latest Results from MEG, PoS (NEUTEL2017) 023.

[45] CMS collaboration, Searches for long-lived charged particles in pp collisions at $\sqrt{s}=7$ and $8 \mathrm{TeV}$, JHEP 07 (2013) 122 [arXiv: 1305. 0491] [INSPIRE].

[46] ATLAS collaboration, Search for the electroweak production of supersymmetric particles in $\sqrt{s}=8$ TeV pp collisions with the ATLAS detector, Phys. Rev. D 93 (2016) 052002 [arXiv: 1509.07152] [INSPIRE].

[47] ATLAS collaboration, Search for squarks and gluinos in final states with jets and missing transverse momentum using $36 \mathrm{fb}^{-1}$ of $\sqrt{s}=13 \mathrm{TeV}$ pp collision data with the ATLAS detector, Phys. Rev. D 97 (2018) 112001 [arXiv:1712.02332] [INSPIRE].

[48] Sld Electroweak Group, Delphi, aleph, SLD, SLD Heavy Flavour Group, OPAL, LEP Electroweak Working Group and L3 collaborations, S. Schael et al., Precision electroweak measurements on the $Z$ resonance, Phys. Rept. 427 (2006) 257 [hep-ex/0509008] [INSPIRE].

[49] M. Chala, Direct bounds on heavy toplike quarks with standard and exotic decays, Phys. Rev. D 96 (2017) 015028 [arXiv: 1705.03013] [INSPIRE].

[50] J. Kawamura, S. Okawa and Y. Omura, Interplay between the $b \rightarrow$ sll anomalies and dark matter physics, Phys. Rev. D 96 (2017) 075041 [arXiv: 1706.04344] [INSPIRE].

[51] K. Kowalska and E.M. Sessolo, Expectations for the muon g-2 in simplified models with dark matter, JHEP 09 (2017) 112 [arXiv:1707.00753] [INSPIRE].

[52] L. Calibbi, R. Ziegler and J. Zupan, Minimal models for dark matter and the muon $g-2$ anomaly, JHEP 07 (2018) 046 [arXiv: 1804.00009] [INSPIRE]. 